\title{
Kinematic and kinetic parameters associated with running in different shoes
}

\author{
Peter J. McNair PhD and Robert N. Marshall* PhD \\ Faculty of Health Studies, Auckland Technical Institute, Auckland, New Zealand, and *Department of Human \\ Movement, The University of Western Australia, Nedlands, Western Australia
}

\begin{abstract}
The characteristics of the midsole were examined in four pairs of running shoes by a materials test. The variables of interest were the peak acceleration, time to peak acceleration and the kinetic energy absorbed. Ten subjects then ran at a recreational jogging pace $\left(3.5 \mathrm{~m} \mathrm{~s}^{-1}\right)$ barefoot and in the shoes. An accelerometer secured to the lower tibia was used to measure the peak acceleration and time to peak acceleration associated with footstrike. Subjects were also videoed and a kinematic analysis was undertaken at the knee and ankle joints. The results from the materials test showed that the shoes differed in their midsole characteristics, however, no significant differences $(P>0.05)$ were observed in the peak acceleration and time to peak acceleration during running in shoes. These variables were significantly greater in the barefoot running condition $(P<0.05)$, as compared with running in shoes. Small and subtle kinematic differences were observed between the barefoot and shoe conditions. It appears that the differences observed between the shoes in the materials test were not sufficient to elicit the kinematic changes observed between the barefoot and shoe conditions. It is suggested that runners operate within a 'kinetic bandwidth' when responding to impact stresses.
\end{abstract}

Keywords: Gait, running, shoes, kinematics, kinetics

During gait, repetitive loading at footstrike generates intermittent impulse forces which are propagated through the musculosketetal system ${ }^{1}$. These impulse forces are gradually attenuated as they travel toward the head by the body's natural shock absorbers such as the heel pad, subchondral bone, articular cartilage, intervertebral discs and menisci ${ }^{2}$. Some researchers $s^{1,3-5}$ have suggested the repetitive nature and magnitude of these impulse forces are associated with musculoskeletal injury. A primary purpose of shoes is to protect the individual from the possible injurious effects of repetitive loading. This can be done in a variety of ways, for instance by reducing the peak acceleration experienced, by increasing the time to peak acceleration or by absorbing a larger proportion of the impact energy. A wide variety of materials and design concepts are used by shoe companies to achieve this purpose.

A number of studies have contributed to our knowledge of impact forces associated with wearing

Address for correspondence: Dr Peter J. McNair, Faculty of Health Studies, Auckland Institute of Technology, Private Bag, Auckland, New Zealand

(C) 1994 Butterworth-Heinemann Ltd 0306-3674/94/040256-05 footwear during gait activities. Light $e t$ al. ${ }^{6}$ compared measurements of tibial accelerations at heelstrike during walking in different footwear and observed that accelerations were significantly higher during walking barefoot or in leather shoes as compared with soft rubber-soled shoes and shoes with viscoelastic inserts. Similarly, Cavanagh et al. ${ }^{7}$ also noted that the initial peak vertical ground reaction force was significantly higher when walking barefoot compared with wearing other types of walking shoes. In contrast to the above researchers, Clarke et al. ${ }^{8}$ observed no difference in the peak ground reaction force associated with footstrike during running in two shoes of different midsole hardness, but noted an increased time to peak force when wearing softer soled running shoes. Hamill and Bates ${ }^{9}$ also reported no significant differences in the vertical ground reaction forces between two pairs of shoes with different midsole characteristics after $420 \mathrm{~km}$ of running, but in contrast to Clarke et al. ${ }^{8}$, no significant differences were observed in the time to peak force of the two shoes. Nigg et al. ${ }^{10}$ and Nigg and Bahlsen ${ }^{11}$ have noted that shoes with very soft midsoles (Shoe Hardness No. 20) have the highest vertical ground reaction forces during running. In this regard, Nigg et al. ${ }^{10}$ commented that a 'bottoming out' effect, where the midsole material is too soft and quickly becomes totally compressed, might be responsible.

The interaction of the runner and the shoe are an important consideration in the development of shoes which best serve to prevent injury. Hence, the purpose of this study was to examine the acceleration and energy absorption characteristics associated with material testing of four shoes with different shock attenuating materials and design concepts, and then to relate these findings to tibial accelerations and sagittal plane kinematics measured during running in these same shoes.

\section{Methods}

Four pairs of shoes with different sole design and shock attenuating materials were examined. All the shoes had a curved, slip last construction, and carbon rubber outsoles. The primary material used in the midsole construction of all the shoes was ethylene vinyl acetate (EVA). However, some of the manufacturers had supplemented this shock absorbing material with other elements and features which 
were believed to enhance the shock attenuation characteristics of the shoes. Hence, the shoes were categorized by the following characteristics: (A) double density EVA with a cantilever outsole; (B) double density EVA; (C) air filled chambers within a double density EVA midsole; (D) encapsulated double density EVA. The shoes could also be differentiated by their price, with a $150 \%$ difference between the most and least expensive shoes.

\section{Material tests}

The procedures used in the material tests were similar to those outlined by Misevich and Cavanagh $^{12}$. A mechanical release system was used to drop a 9-kg mass from a height of $5 \mathrm{~cm}$. The vertical velocity of the mass at impact was approximately $1.0 \mathrm{~m} \mathrm{~s}^{-1}$. These parameters were chosen to simulate as closely as possible the accelerations and mass experienced during recreational jogging. The impact head was circular, $2.5 \mathrm{~cm}$ in diameter with a $9.0-\mathrm{cm}$ radius of curvature. Shoes were firmly clamped to prevent movement occurring at the time of impact. Each shoe type was subjected to impact 20 times.

The variables of interest in the material tests were the peak acceleration, the time to peak acceleration and the kinetic energy absorbed by the shoe. The kinetic energy (KE) of the mass at its initial impact was determined by the formula $\mathrm{KE}=1 / 2 m v^{2}$, where $m$ is the mass and $v$ is the velocity of the mass at impact. Typically, the mass would rebound after impact and a knowledge of the time period between impacts allowed a calculation of the height attained in the rebound phase. With this information, the kinetic energy at the time of the second impact was calculated. The kinetic energy absorbed was determined from a knowledge of the kinetic energy associated with these impacts.

\section{Treadmill running}

In the running section of the study, ten men (mean(s.d) age 25(5) years; mean(s.d.) mass $75(6) \mathrm{kg}$ who ran regularly for fitness and/or club competition were used as subjects. All subjects were heelstrikers. The procedures involved securing a Phillips 20-g accelerometer (model PC9367/20; Phillips, Nijgmegan, Netherlands) to the lower medial aspect of the tibia. The method of attachment involved first wrapping double sided adhesive tape around the lower tibia. The active axis of the accelerometer was then aligned with the longitudinal axis of the tibia and firmly applied to the tape. Zinc oxide tape was then wrapped around the tibia over the accelerometer as firmly as possible without causing the subject undue discomfort. Accelerometer signals were amplified by a Kyowa dynamic strain amplifier (model DPM-110A; Kyowa, Tokyo, Japan) and relayed to a computer-based data acquisition package sampling at $1000 \mathrm{~Hz}$. Peak accelerations were measured and averaged across subjects and trials.

Subjects ran on a motor driven treadmill at $3.5 \mathrm{~m} \mathrm{~s}^{-1}$. All subjects had experienced treadmill running before testing. For all footwear conditions, subjects ran for $5 \mathrm{~min}$ in the shoes being tested before the collection of data. Video data were collected at 200 frames per second. The camera was secured on a tripod so that the focal axis of the lens was perpendicular to the plane of motion, and the camera-subject distance $(5.0 \mathrm{~m})$ was large enough to minimize perspective error. Adhesive retroreflective markers were placed either on the skin or the shoe over the following anatomical landmarks: fifth metatarsal head; lateral calcaneus; lateral malleolus; lateral epicondyle of the femur; and the greater trochanter of the femur. For the shoe tests, the anatomical landmarks were determined by palpation through the shoe material. A Motion Analysis Flextrack system (Motion Analysis, Santa Rosa, California, USA) was used to digitize eight strides of data for each subject. Segment angle data were then determined for the foot, leg and thigh. The knee and ankle joint angles at footstrike and the net amount of stance and swing phase flexion (footstrike angle minus most flexed position in stance and swing respectively) were recorded. These discrete values were averaged over eight strides and a repeated measures analysis of variance was used to establish the statistical significance of the data. The knee and ankle angle time-series data for 80 trials of each shoe (ten subjects $\times$ eight strides) were then plotted (Figures 6 and 7). One standard error either side of the mean was also included for each shoe.

Subjects were also asked for their comments regarding the shock attenuating properties of the shoes.

\section{Results}

\section{Material tests}

The mean peak accelerations associated with each shoe type from the materials testing are presented in Figure 1. Standard error bars of the mean are shown, but in general they are smaller than the figure symbols for many of the tests. There were significant differences between all shoes $(P<0.05)$ and approximately a $17 \%$ range between the shoe with the lowest (A) and highest (D) peak acceleration. The mean times to peak acceleration associated with each shoe

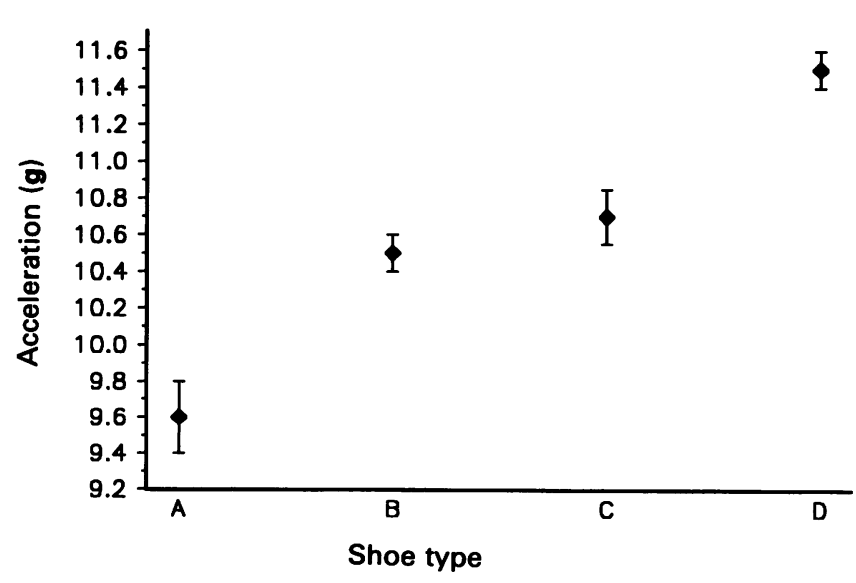

Figure 1. Mean(s.e.) peak accelerations associated with shoes during the material test 
type are presented in Figure 2. Significant differences $(P<0.05)$ were observed between all times except between shoes $B$ and $D$, with approximately a $30 \%$ range between the longest $(\mathrm{A})$ and shortest $(\mathrm{C})$ times. The mean kinetic energy absorbed by the shoes is

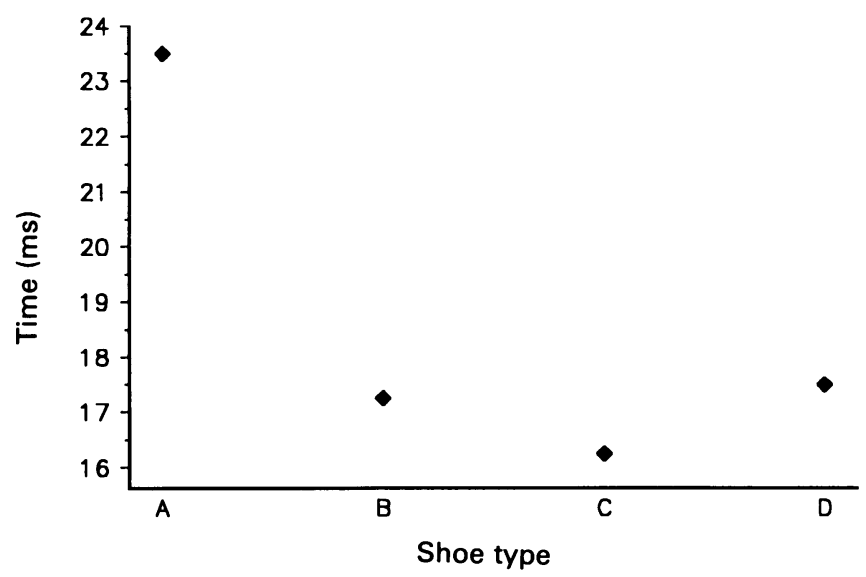

Figure 2. Mean(s.e.) time to peak acceleration associated with footwear during the material test

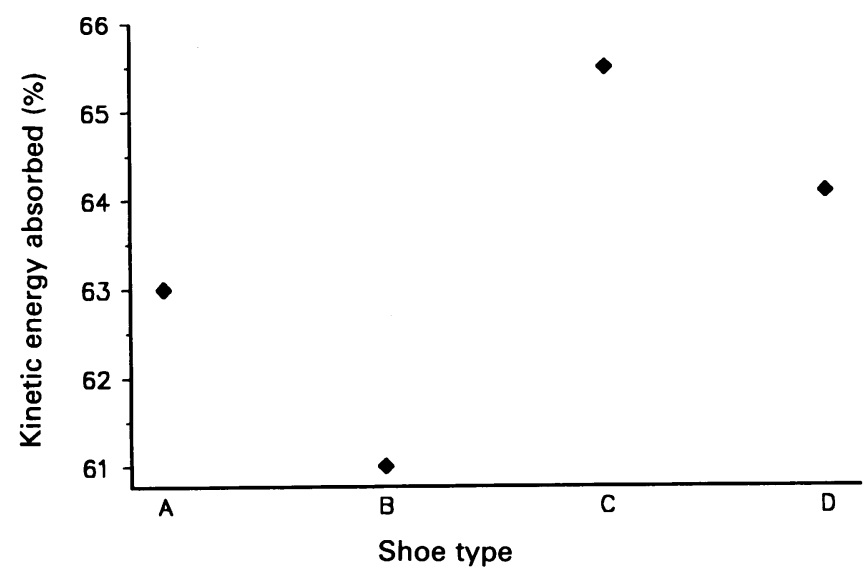

Figure 3. Mean(s.e.) absorbed kinetic energy associated with footwear during the material test

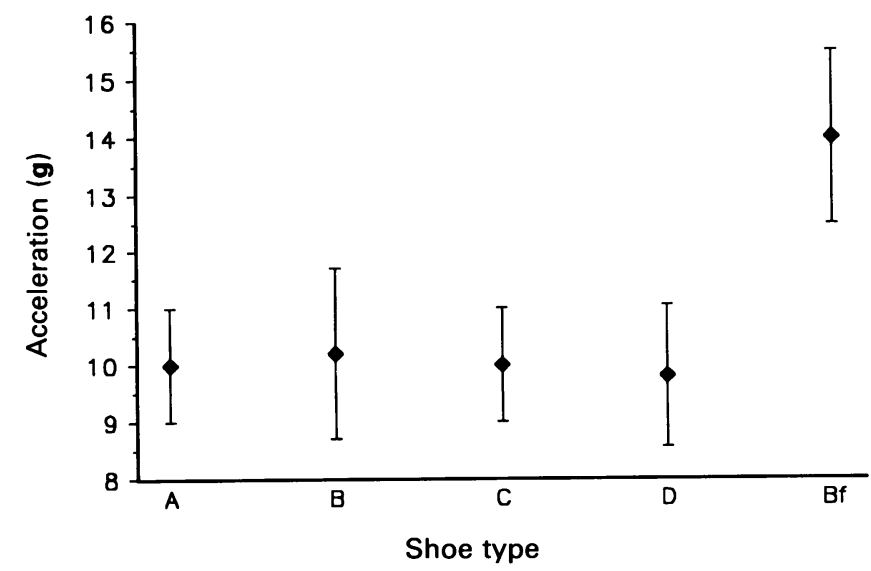

Figure 4. Mean(s.e.) peak tibial accelerations during running in different footwear: $\mathrm{Bf}$, barefoot running presented in Figure 3. There were significant differences $(P<0.05)$ between all shoes, with a difference of $4 \%$ between the most (C) and the least (B) kinetic energy absorbed.

\section{Treadmill running}

The mean accelerations measured at the tibia during treadmill running are presented in Figure 4. Average values between 9 and $10 \mathrm{~g}$ are seen for all shoe types over subjects and trials. There were no significant differences between shoes, however, the peak acceleration recorded during barefoot running was significantly higher than that observed for all shoe types. The variability of the data was similar across the different shoe types and the barefoot running.

The times to peak acceleration are presented in Figure 5. While there were no significant differences between shoes, the time to peak acceleration recorded during barefoot running was significantly lower $(P<0.05)$ than those observed for shoes. The time of peak acceleration was approximately $34.5 \mathrm{~ms}$ across shoes, while for barefoot running it was $48 \%$ lower at $18 \mathrm{~ms}$.

The mean(s.e.) for the angular displacement of the knee during the stride cycle is presented in Figures $6 a$ and $b$. The results suggest that the knee joint motion throughout the stride cycle was relatively invariant across both shoes and the barefoot condition, although in parts of the stride cycle some subtle differences were consistently observed between the barefoot and shoe conditions. For instance, the net amount of flexion in stance was slightly less $(P<0.05)$ when running barefoot. At toe-off (at approximately $40 \%$ of the stride cycle) the knee is slightly more flexed when running barefoot. In the swing phase the maximum knee flexion is greater when running in shoes compared with running barefoct $(P<0.05)$.

The mean(s.e.) for the angular displacement of the ankle joint during the stride cycle is presented in Figures $7 a$ and $b$. It was apparent that throughout the stride cycle the subjects maintained the ankle joint in a more plantar flexed position $(P<0.05)$ in the barefoot condition compared with running in the shoes. However, the net amount of dorsiflexion or

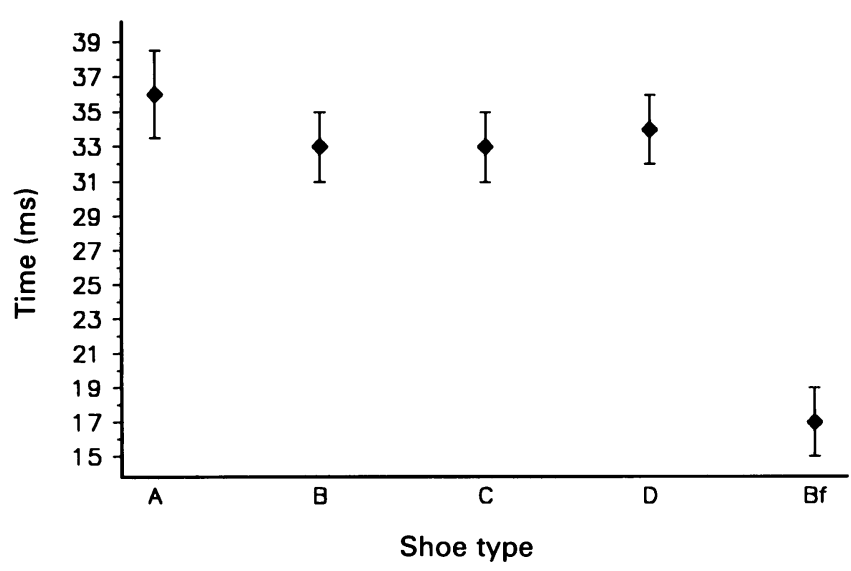

Figure 5. Mean(s.e.) time to peak acceleration for different footwear during running 


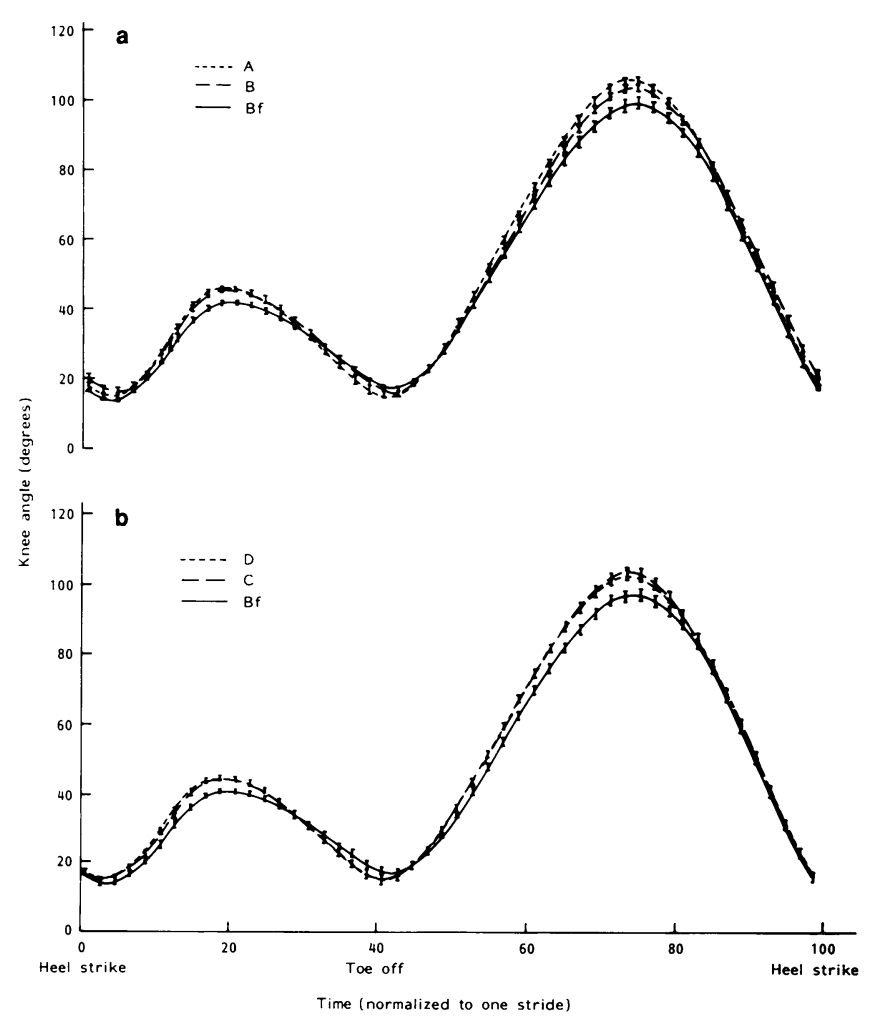

Figure 6. a, b Mean(s.e.) angular displacement of the knee joint through the stride cycle

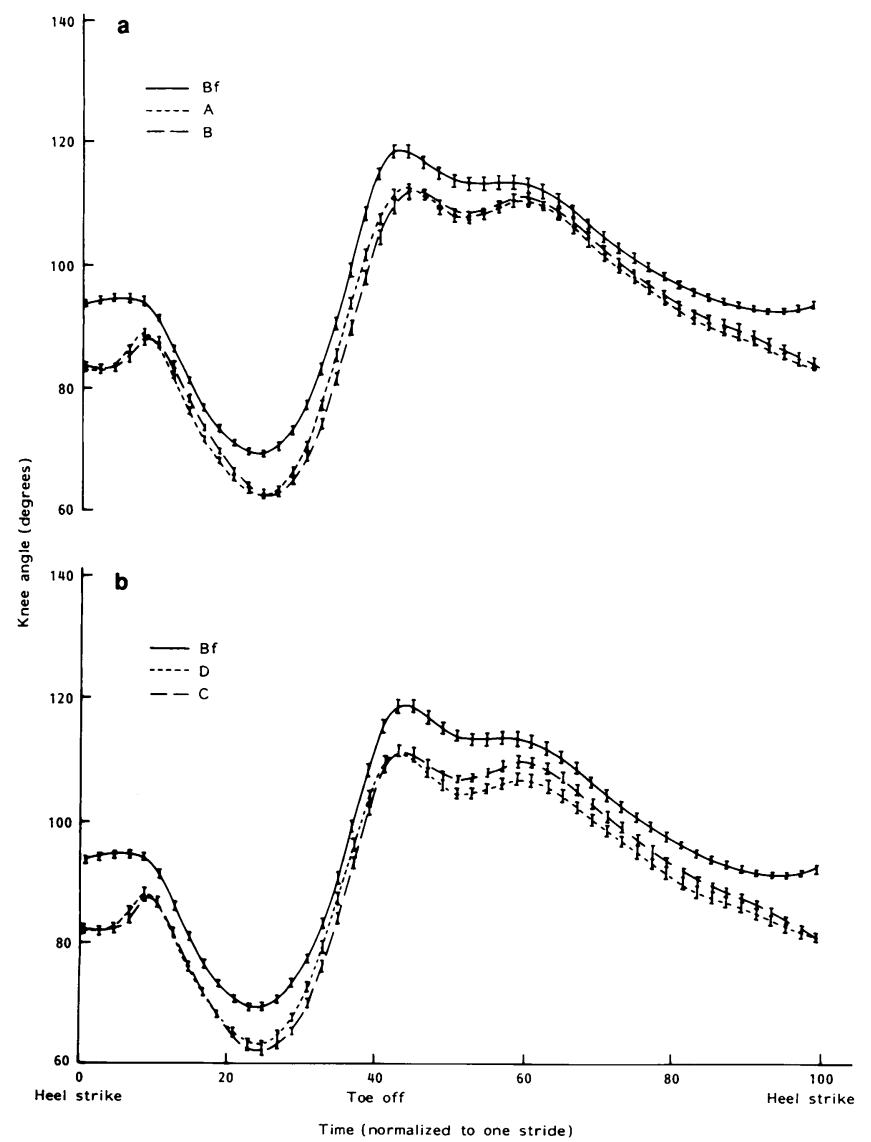

Figure 7. a, b Mean(s.e.) angular displacement at the ankle joint through the stride cycle plantar flexion was similar $(P>0.05)$ between the shoe and barefoot conditions.

\section{Discussion}

Accelerometers have been used by many researchers ${ }^{1,3,4,6}$ to measure the magnitude and nature of the transient impulse forces associated with footstrike. The mean peak accelerations measured at the lower medial tibia were within the range of those reported by previous investigators ${ }^{6,13}$.

In the current study all the manufacturers had chosen to use EVA as the primary material of the midsole component of the shoe. Additionally they had chosen to modify this component with various design concepts to improve the shock attenuation qualities of the shoe. Differences were observed between the shoes in the material tests, particularly in the peak accelerations, whereas in the time to peak acceleration, shoe A had a considerably longer time to peak and the other shoes all peaked at similar times. Similarly, the kinetic energy absorbed differed only by $4 \%$ between those shoes used in the running test. With regard to shock attenuation, if the shoes could be ranked according to their ability to lessen the stresses associated with impact it was apparent that no one shoe was consistent in all the tests, although shoe A was the most effective in decreasing the peak acceleration and increasing the time to peak acceleration

The validity of materials testing to investigate the impact characteristics of a shoe has been questioned by $\mathrm{Nigg}^{14}$. He showed that the results of such tests are influenced by the mass of the impacting object together with its vertical velocity and area of contact at impact with the shoe. In our study, the impact mass, velocity and impact head characteristics were similar to those used by other researchers, which were based on actual data from runners. The peak accelerations recorded in this study were similar to those observed during running, however the time to peak accelerations were considerably less than those recorded during the jogging test. This difference may be related to the single mass drop model used, and limits the direct applicability of these results to the human-shoe interaction.

However, in the materials testing, the shoe which could be regarded as performing the 'best' with regard to minimizing the effect of impact forces (shoe A) was also the shoe which subjects unanimously regarded as the best shoe with regard to reducing the perceived impact stresses. This shoe was also the most expensive shoe. As would be expected, subjects also stated that they thought that the barefoot condition was significantly less shock attenuating than any of the shoe conditions. This suggested that the runners were not altering their running style in response to this perception, and indeed the higher peak tibial accelerations and lower times to peak acceleration observed during barefoot running provided support for this notion. Similarly the minimal differences in knee joint kinematics observed among shoes and between shoes and bare feet provided further support for the suggestion that the shoes 
acted simply as a buffer to the peak forces associated with footstrike, and the motor programme generating the stereotypical pattern of the lower limb during gait was unaffected by footwear. However, the results pertaining to the ankle joint do not appear to support this conjecture as they show that the position of the foot was more plantar-flexed during barefoot running throughout the stride cycle. The significance of this position is difficult to speculate upon. Nevertheless, the net amount of plantar and dorsiflexion occurring in both stance and swing phases was similar to that observed for running in shoes, providing further support for the invariance of the motor programme associated with running.

An alternative suggestion is that there are probably numerous combinations of joint and muscle activity, of which the individual parameters need only alter slightly to produce considerable changes in the kinetics associated with footstrike. For instance, increasing the knee angle at footstrike, decreasing the vertical velocity of the foot at landing and increasing gastrocnemius activity have been shown to be effective ways for achieving this purpose ${ }^{15,16}$. The modifications to muscle and joint activity when changing from running in a 'good' running shoe to barefoot are probably much less subtle than those changes which occur when changing between a number of running shoes. Hence, it may be that some of the numerous subtle joint and muscle activity modifications which occurred to produce kinetic changes may not have been measured, or that the modifications were individually of such a subtle nature that they were within the measurement error of the monitoring system used.

In conclusion, the verbal comments of subjects concerning shock attenuation would suggest that we can readily perceive differences in a shoe's shockabsorbing capabilities. Whether the body responds to these perceptions is not clear, since the tibial accelerations for barefoot running were higher than those recorded during running in footwear, and many of the kinematics parameters measured remained unchanged. It would appear that runners prefer to operate within an impact acceleration 'bandwidth' as evidenced by the similarity of tibial accelerations across shoes, and by subtle but distinct differences in the ankle and knee kinematics.

It may be that the body responds to shoe wear in many instances not just by altering one or two parameters remarkably, but more likely by a number of subtle changes to the lower limb muscle and joint activity. As such the neuromotor system is acting in a sensitive manner and we do not yet have a good understanding of the numerous permutations of muscle and joint activity that may facilitate the attenuation of impact forces.
The practical implication of our findings is that the recreational runner who is buying a new pair of shoes need not be overwhelmed by the numerous different shock absorption materials and design concepts available, since it appears that at this time there are few differences to be observed between shoes with regard to shock attenuation during slow-speed running.

\section{References}

1 Chu MC, Yazdani-Ardakani S, Gradiser IA, Askew MJ. An in-vitro simulation study of force transmission along the lower skeletal extremity. J Biomech 1986; 19: 979-87.

2 Wosk J, Voloshin A. Wave attenuation in skeletons of young healthy persons. J Biomech 1981; 14: 261-7.

3 Voloshin A, Wosk J. Shock absorbing capacity of the human knee (in-vivo properties). Proceedings of the Canadian Society of Biomechanics. Waterloo, Canada: University of Waterloo Press, 1980: 27-9.

4 Voloshin A, Wosk J, Brull M. Force wave transmission through the human locomotor system. J Biomech Eng 1981; 103: 48-50.

5 MacLellan GE, Vyvyan B. Management of pain beneath the heel and Achilles tendinitis with visco-elastic heel inserts. $\mathrm{BrJ}$ Sports Med 1981; 15: 117-21.

6 Light LH, McLellan GE, Klenerman L. Skeletal transients on heelstrike in normal walking with different footwear. J Biomech 1980; 13: 477-80.

7 Cavanagh PR, Williams KR, Clarke TE. A comparison of ground reaction forces during walking barefoot and in shoes. In: Moreck K, Fidelus K, Kedzior K, Wit A, eds. Biomechanics VII-B. Baltimore, Ohio, USA: Baltimore University Park Press, 1981: 151-6.

8 Clarke TE, Frederick EC, Cooper LB. Effects of shoe cushioning upon ground reaction forces in running. Int $J$ Sports Med 1983; 4: 247-51.

9 Hamill J, Bates BT. A kinetic evaluation of the effects of in-vivo loading on running shoes. J Orthop Sports Phys Ther 1988; 10: 47-53.

10 Nigg BM, Bahlsen AH, Denoth J, Luethi SM, Stacoff A. Factors influencing kinetic and kinematic variables in running. In: Nigg BM, ed. Biomechanics of Running Shoes. Champaign, Illinois, USA: Human Kinetics, 1986; 139-59.

11 Nigg BM, Bahlsen HA. Influence of heel flare and mid-sole construction on pronation, supination, and impact forces for heel-toe running. Int J Sport Biomech 1988; 4: 205-19.

12 Misevich K, Cavanagh P. Material aspects of modelling shoe/foot interaction. In: Frederick EC, ed. Sports Shoes and Playing Surfaces. Champaign, Illinois, USA: Human Kinetics, 1984: 47-75.

13 Clarke TE, Cooper LB, Clark DE, Hamill CL. The effect of increasing running speed upon peak shank deceleration during ground contact. In: Winter D, Norman R, Wells RA, Hayes K, Patla A, eds. Biomechanics IX-B. Champaign, Illinois, USA: Human Kinetics, 1985: 101-5.

14 Nigg BM. The validity and relevance of tests used for the assessment of sports surfaces. Med Sci Sports Exerc 1990; 22: 131-9.

15 Nigg BM, Denoth J, Kerr B, Luethi S, et al. Load, sports shoes and playing surfaces. In: Frederick EC, ed. Sports Shoes and Playing Surfaces. Champaign, Illinois, USA: Human Kinetics, 1984: 1-23.

16 Pratt D. Mechanisms of shock attenuation via the lower extremity during running. Clin Biomech 1989; 4: 51-7. 